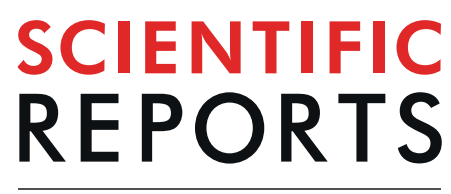

\title{
SureSelect targeted enrichment, a new cost effective method for the whole genome sequencing of Candidatus Liberibacter asiaticus
}

\begin{abstract}
Weili Cai ${ }^{1,2}$, Schyler Nunziata $^{1}$, John $\operatorname{Rascoe}^{1} \&$ Michael J. Stulberg $\mathbb{D}^{1 *}$
Huanglongbing (HLB) is a worldwide deadly citrus disease caused by the phloem-limited bacteria 'Candidatus Liberibacter asiaticus' (CLas) vectored by Asian citrus psyllids. In order to effectively manage this disease, it is crucial to understand the relationship among the bacterial isolates from different geographical locations. Whole genome sequencing approaches will provide more precise molecular characterization of the diversity among populations. Due to the lack of in vitro culture, obtaining the whole genome sequence of CLas is still a challenge, especially for medium to low titer samples. Hundreds of millions of sequencing reads are needed to get good coverage of CLas from an HLB positive citrus sample. In order to overcome this limitation, we present here a new method, Agilent SureSelect ${ }^{\mathrm{X} \mathrm{HS}}$ target enrichment, which can specifically enrich CLas from a metagenomic sample while greatly reducing cost and increasing whole genome coverage of the pathogen. In this study, the CLas genome was successfully sequenced with $99.3 \%$ genome coverage and over $72 X$ sequencing coverage from low titer tissue samples (equivalent to 28.52 Cq using Li 16 S qPCR). More importantly, this method also effectively captures regions of diversity in the CLas genome, which provides precise molecular characterization of different strains.
\end{abstract}

Huanglongbing (HLB), or citrus greening, is a devastating citrus disease caused by phloem-restricted gram-negative bacteria 'Candidatus Liberibacter' $\mathrm{spp}^{1,2}$. There are three $\alpha$-proteobacteria associated with HLB: "Candidatus Liberibacter asiaticus", "Ca. Liberibacter americanus" and "Ca. Liberibacter. africanus"1,3. 'Ca. Liberibacter asiaticus' (CLas) is the most widespread and is the only species associated with the disease in the United States (U.S.) ${ }^{4}$. CLas associated HLB was first found in Florida in early September, $2005^{5}$ and was vectored by the Asian citrus psyllid (Diaphorina citri), which had been introduced into Florida in the late 1990s. The disease has since been identified in multiple states (USDA APHIS Citrus Greening Quarantine map, https://www. aphis.usda.gov/plant_health/plant_pest_info/citrus_greening/downloads/pdf_files/nationalquarantinemap.pdf).

Effective disease managing efforts require a greater understanding of the causal agents, which can be achieved through whole genome sequencing. The genetic identity of strains found in new locations or with varying aggressiveness can help inform the effectiveness of quarantine programs and provide researchers with data to search for virulence-associated genetic elements. Identifying aggressive strains might impact future management practices if zero tolerance policies are no longer applicable. Providing strain identification can help inform pathogen dissemination.

Whole genome sequencing can provide precise molecular characterization of the diversity among CLas populations. Currently, conserved genomic loci, such as the $16 \mathrm{~S}$ rRNA gene, are used to define the CLas species but lack the genetic variation to differentiate strains $s^{6,7}$. Population variation studies using PCR to amplify several genomic loci or short tandem repeats regions might not provide sufficiently high resolution to differentiate all strains from multiple locations ${ }^{8-12}$. A pan-genome comparative approach could provide enough genetic variation for high strain resolution, but sequencing CLas genomes has been historically difficult. The first CLas genome sequence was released in 2009 , isolated from a single infected psyllid ${ }^{13}$, and in nearly 10 years since there have

\footnotetext{
${ }^{1}$ Science and Technology, Plant Protection and Quarantine, Animal and Plant Health Inspection Service, United States Department of Agriculture, Beltsville, Maryland, United States of America. ${ }^{2}$ Department of Entomology and Plant Pathology, North Carolina State University, Raleigh, North Carolina, United States of America. *email: Michael. Stulberg@usda.gov
} 


\begin{tabular}{|l|l|l|l|l|l|l|l|}
\hline \multirow{2}{*}{ ID } & Enriched & Total Reads & $\begin{array}{l}\text { Total Aligned } \\
\text { Reads }\end{array}$ & $\begin{array}{l}\text { Alignment } \\
\text { Rate (\%) }\end{array}$ & $\begin{array}{l}\text { Fold } \\
\text { Enrichment }\end{array}$ & $\begin{array}{l}\text { Coverage } \\
(\%)\end{array}$ & $\begin{array}{l}\text { Sequence } \\
\text { Coverage }\end{array}$ \\
\hline \multirow{2}{*}{ LHCA20 } & Yes & $2,634,608$ & $1,178,690$ & 44.74 & \multirow{2}{*}{497} & 99.70 & $295 \mathrm{X}$ \\
\cline { 2 - 5 } & No & $6,109,250$ & 5,494 & 0.09 & & 64.90 & $1.3 \mathrm{X}$ \\
\hline \multirow{2}{*}{ LHCA22 } & Yes & $3,486,268$ & $2,121,784$ & 60.86 & \multirow{2}{*}{4631} & 99.80 & $505 \mathrm{X}$ \\
\cline { 2 - 5 } & No & $7,069,576$ & 929 & 0.01 & & 16.80 & $0.2 \mathrm{X}$ \\
\hline \multirow{2}{*}{ LHCA26 } & Yes & $3,299,660$ & 691,607 & 20.96 & \multirow{2}{*}{45113} & 99.40 & $165 \mathrm{X}$ \\
\cline { 2 - 5 } & No & $7,102,694$ & 33 & 0.00 & & 0.50 & $0 \mathrm{X}$ \\
\hline \multirow{2}{*}{ LHCA28 } & Yes & $3,277,008$ & 328,121 & 10.01 & \multirow{2}{*}{19165} & 99.30 & $72 \mathrm{X}$ \\
\cline { 2 - 5 } & No & $4,019,510$ & 21 & 0.00 & & 0.40 & $0 \mathrm{X}$ \\
\hline \multirow{2}{*}{ SGCA20 } & Yes & $1,467,124$ & $1,089,745$ & 74.28 & \multirow{2}{*}{1068} & 99.50 & $258 \mathrm{X}$ \\
\cline { 2 - 5 } & No & $6,888,648$ & 4,790 & 0.07 & & 60.60 & $1.2 \mathrm{X}$ \\
\hline \multirow{2}{*}{ SGCA22 } & Yes & $3,093,380$ & $2,302,812$ & 74.44 & \multirow{2}{*}{4444} & 99.50 & $551 \mathrm{X}$ \\
\cline { 2 - 4 } & No & $7,450,500$ & 1,248 & 0.02 & & 21.70 & $0.3 \mathrm{X}$ \\
\hline
\end{tabular}

Table 1. Alignment summary of CLas sample reads to the genome of CLas strain Psy62 using bowtie2.

been only 14 additional CLas genomes deposited to NCBI (only five are complete). The released CLas genomes were obtained from either highly infected psyllids or citrus samples (equivalent to 18 to 23 Cq using Li 16S qPCR $)^{14-17}$ because the whole genome sequence of CLas can only be obtained using metagenomic sequencing, due to the lack of in vitro culture. Such high pathogen titer samples are needed because a low percentage of sequencing reads belonging to CLas are present in a metagenomic sample, primarily because of large genome size difference between pathogen and host and relative low copy number of pathogen DNA. Hundreds of millions of sequencing reads are needed to get good CLas genome coverage from an infected citrus sample, making CLas genome sequencing challenging and costly ${ }^{18}$. Additionally, to study the impact of strain diversity in CLas epidemiology, it is important to include more geographic locations, and newly infected samples often carry a much lower pathogen titer than the successfully sequenced samples. Thus a targeted genome enrichment method may be useful and necessary.

Targeted genome enrichment specifically enriches sequences of interest within a heterogeneous mixture of DNA samples. For target selection, pre-designed probes are added to the mixed genomic DNA extracts and capture their complimentary DNA sequences through complimentary hybridization, allowing the uncaptured DNA to be removed during wash steps. With positive target selection, the probe-bound DNA is eluted and collected for further NGS application, and often has much higher target DNA concentration than the original input samples $^{19,20}$. This method has been widely used to capture and enrich targeted DNA from complex biological samples, but is not commonly used to recover plant pathogens from a plant host background ${ }^{21-23}$.

In this study, we assess the ability of a target enrichment method, Agilent SureSelect ${ }^{\text {XT HS }}$ (hereafter referred to as SureSelect), to enrich CLas genomic DNA from infected citrus genomic DNA, and in turn greatly reduce the cost and increase the coverage and reliability of whole genome sequencing.

\section{Results}

Genome alignment and target enrichment. Target enrichment efficiency was estimated by aligning trimmed and quality filtered reads to the CLas strain Psy62 reference genome and comparing alignment rate between enriched and non-enriched samples (Table 1). After trimming and filtering, $40-50 \%$ of the enriched reads were discarded due to insufficient read length and suspected probe contamination, while less than $5 \%$ of non-enriched reads were discarded (Table S3). Without enrichment, LHCA-20 and SGCA-20, the highest pathogen concentration samples, had genome coverage of 65 and $60 \%$, respectively, both with $1 \mathrm{x}$ depth of coverage (Table 1). After SureSelect enrichment, both of these samples had 99\% genome coverage with at least 250X depth of coverage. Enriched samples with the lowest pathogen concentration had $99 \%$ genome coverage and at least $70 X$ sequence coverage. Only small portions of the genome were poorly covered, with more than $90 \%$ of the regions showing a depth of coverage of at least $20 \mathrm{X}$ across all samples (Fig. 1). In general, the same regions were not always missing, with only $\sim 2 \mathrm{~kb}$ shared sites missing across samples. Of the seven shared sites missing across samples, four were in prophage regions that could reflect sequence diversity, and the remaining three regions only totaled approximately $200 \mathrm{bp}$. Pathogen DNA is enriched from 500- to 45,000-fold compared to non-enriched samples. All these results suggest that Agilent SureSelect XT HS target enrichment can effectively capture target DNA from complex CLas samples and significantly increase the pathogen DNA ratio.

Prophage and genome diversity analysis. Next, we assessed how well enrichment captures the genome diversity of different strains. The most divergent region of the CLas genome is the prophage region, where strains can contain one to three prophages, with three prophage types known to date. For non-enriched samples, too few reads aligned to prophage reference sequences to estimate prophage type. Enriched samples, however, had enough reads to align samples to SC1, SC2 and JXGC3 prophage reference sequences. Each LHCA sample contained prophages SC1 and SC2, while SGCA samples contained only SC1 (Fig. 2). This pattern was consistent across different concentrations of the same strain.

To further analyze the repeatability and specificity of this method, we identified and compared the SNPs of these two strains at different Cq values. SNPs were determined based on the alignment profile to Psy62. More 
a.

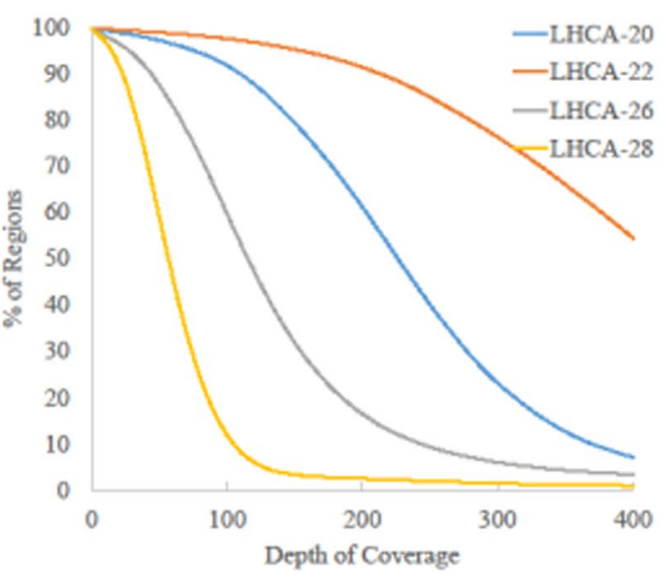

b.

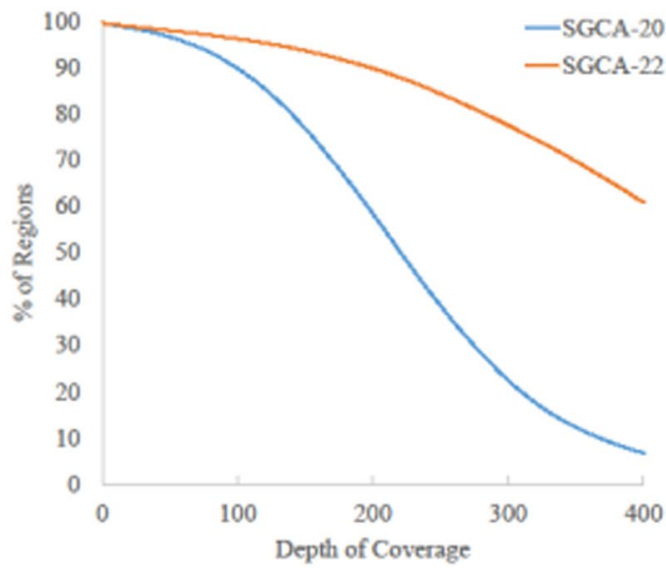

Figure 1. Percentage of bases covered across fixed depths of coverage based on reference guided assemblies and estimated with samtools depth. (a) LHCA samples at different Cq values: Cq 20 (blue), Cq 22 (red), Cq 26 (gray), Cq 28 (yellow). (b) SGCA samples at different Cq values: Cq 20 (blue), Cq 22 (red).

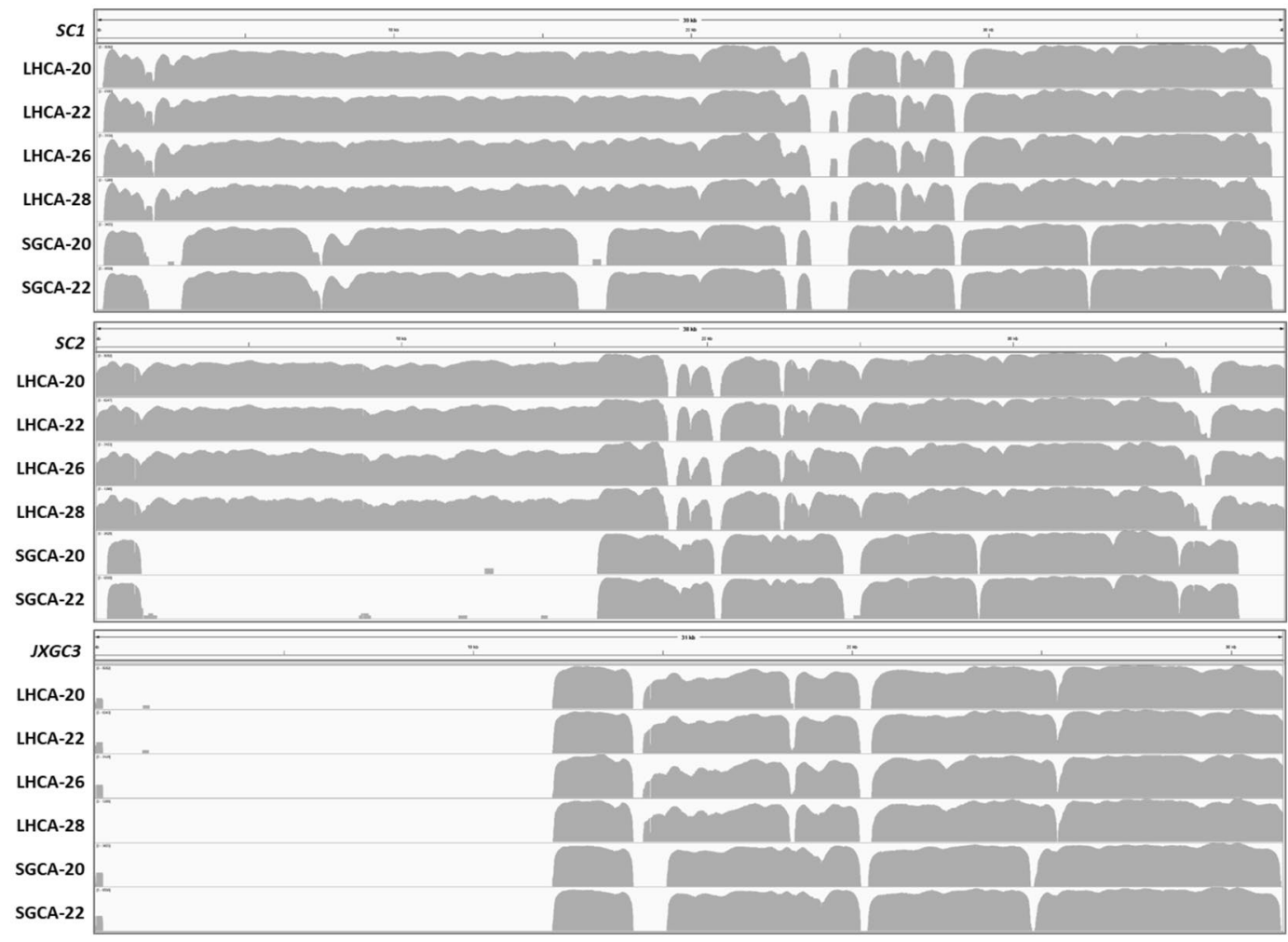

Figure 2. Profiles of CLas MiSeq reads mapping in reference to prophage SCI, SC2 and JXGC-3. Names of CLas samples were listed on the left. Reference prophage genome sequences were at the top. For each CLas samples, gray graphs represent read coverage in log scale. The alignment is generated using bowtie 2 plugged in Geneious v 10.2.4, and visualized in Integrated Genome Viewer v2.4.10.

than $90 \%$ of SNPs were common between two high titer LHCA and SGCA samples, LHCA20/ LHCA22 and SGCA20/SGCA22 (Fig. 3 and Table S4). Less than 45\% of SNPs in LHCA were identified in SGCA samples, suggesting this enrichment method does not change the pan-genome variability.

Phylogenetic analysis. We estimated phylogenies of all samples along with 11 available reference genomes, using both a SNP and pan-genome approach. The SNP tree clearly shows the separation of LHCA and SGCA strains (Figs. 4 and 5). The two SGCA strain samples are clustered together and most closely related to the previously reported SGCA strain, SGCA5. All four LHCA samples are also clustered together. The LHCA strain 
SGCA20

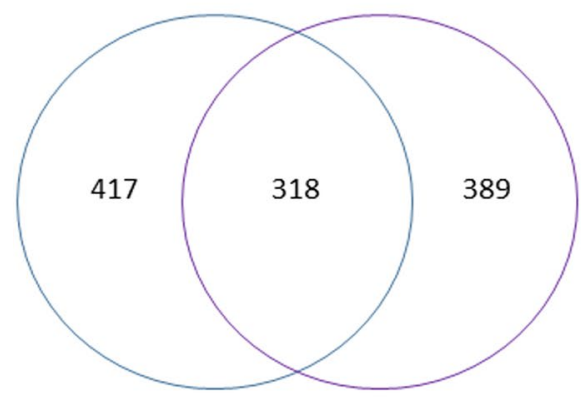

SGCA20

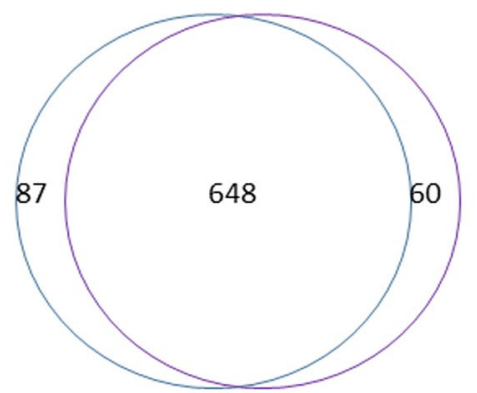

SGCA22

LHCA22

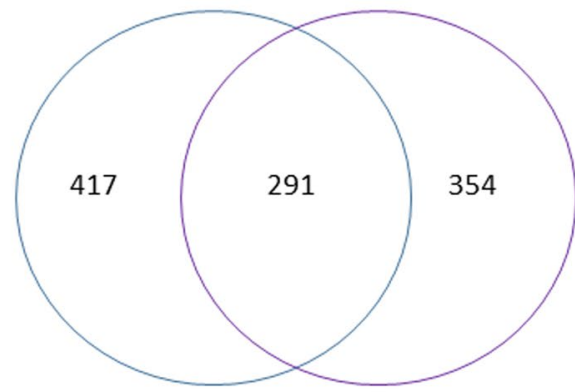

LHCA20

LHCA22

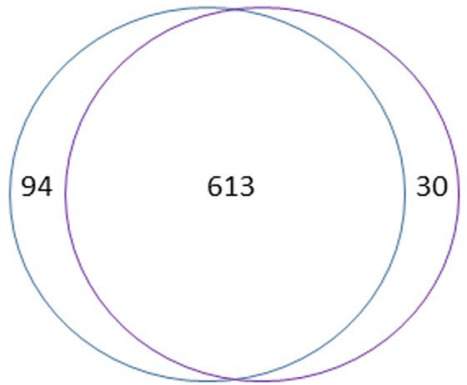

Figure 3. Venn diagrams show the overlapping of SNPs (single nucleotide polymorphisms) from different samples. The number in each circle represents the number of SNPs between the different comparisons. The overlapping number stands for the same SNPs identified between the different comparisons and the nonoverlapping numbers specify the unique SNPs to each sample. SNPs were determined using Samtools v1.7.

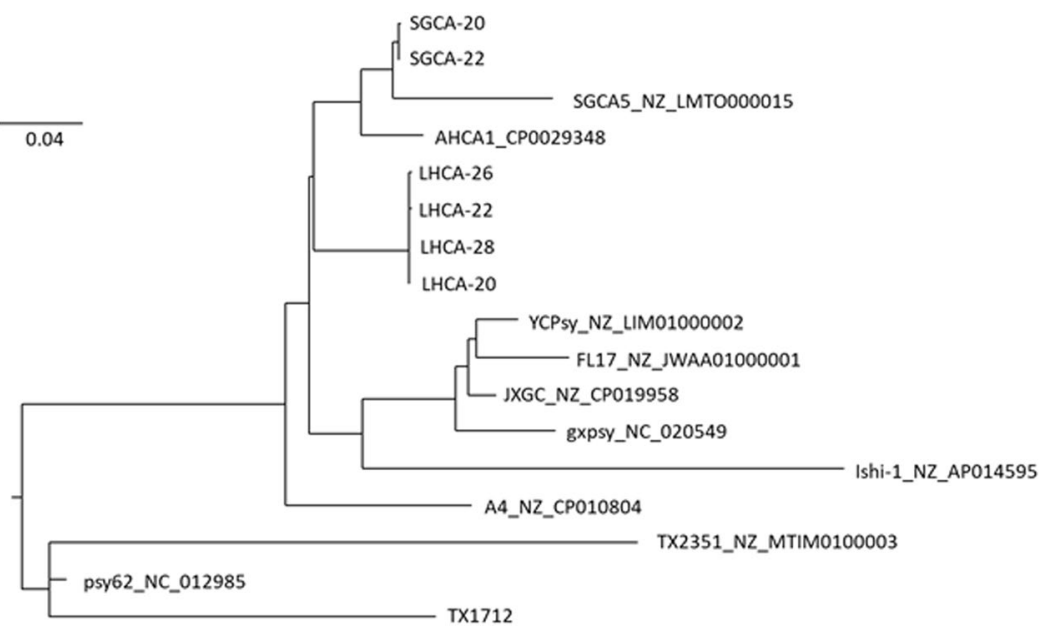

Figure 4. Phylogenic tree (ML midpoint rooted tree) of 849 core SNVs of "Candidatus Liberibacter asiaticus" strains generated with Rax Maximum Likelihood method. SGCA (20 and 22) and LHCA (26,22,28, and 20) were all sequenced in this study. All other genomes were obtained from NCBI. Trees were generated using RaxML v8.2.10 and visualized using FigTree v1.4.3.

clusters most closely to the other reported California strains, AHCA1 and SGCA5, however it does form its own distinct clade from those strains too. The pan-genome phylogenetic tree based on core genes also demonstrates a similar branching pattern.

\section{Discussion}

Over the past ten years, NGS (next generation sequencing) has been widely applied to identity pathogens, characterize genetic variants, and provide a molecular basis for building additional diagnostic tools. However, NGS technology has significant limitations when performing pathogen diagnostics in complex metagenomic samples. Without special enrichment, NGS can rarely detect low copy number pathogen sequences from complex samples 


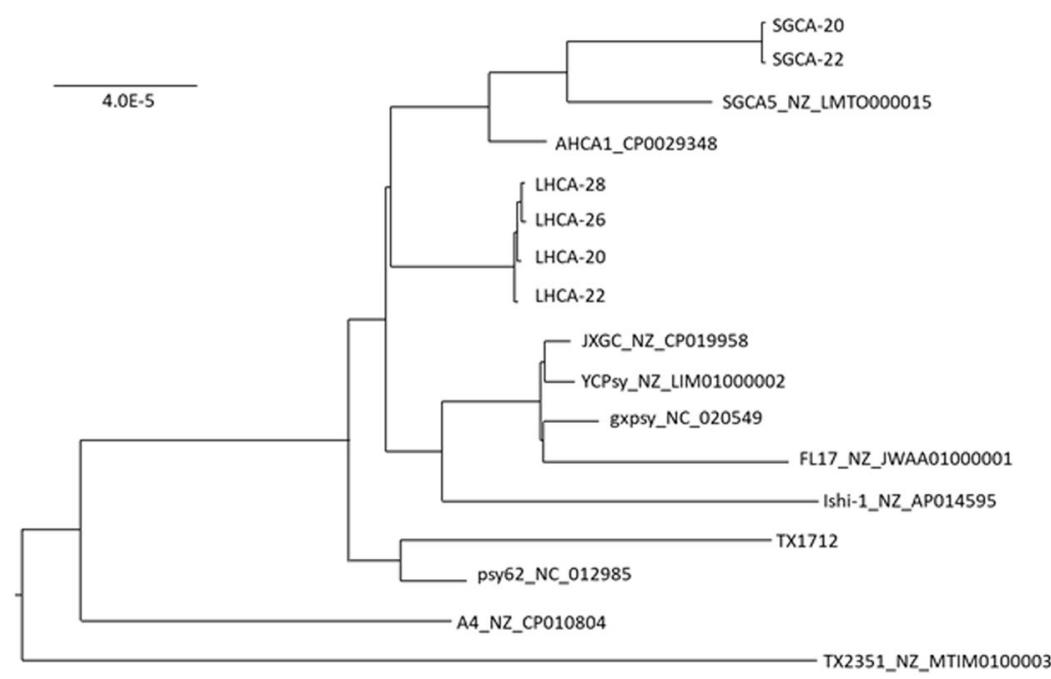

Figure 5. Phylogenic tree (ML midpoint rooted tree) of 935 core genes of "Candidatus Liberibacter asiaticus" strains, generated with Rax Maximum Likelihood method. All other genomes were obtained from NCBI. Trees were generated using RaxML v8.2.10 and visualized using FigTree v1.4.3.

due to low pathogen/host nucleic acid ratio. Prior to this work, obtaining a CLas whole genome sequence was a challenge. Nearly all draft genomes come from highly infected citrus or psyllids (usually with a Cq value lower than 23 using Li 16S qPCR), which limits strain diversity and epidemiology studies since not all samples can be sequenced reliably.

Researchers have used enrichment strategies to increase the number of target reads in sequencing. Previously, the NEBNext microbiome DNA enrichment kit coupled with the REPLI-g amplification kit was used to successfully sequence the HHCA genome from an infected lemon tree with $175 \mathrm{pg}$ of CLas DNA per $\mu \mathrm{l}$ (roughly equivalent to $\mathrm{Cq} 23-24$ using $\mathrm{Li} 16 \mathrm{~S} \mathrm{qPCR}^{6}$ ). This negative target subtraction coupled with microbial enrichment technique still required 78 million total reads to produce $10 \mathrm{X}$ genome coverage after assembly ${ }^{24}$. Hence, non-target enrichment of samples still makes CLas genome sequencing quite difficult and costly, and is not suitable for sequencing low titer samples (e.g. Li Cq 26 and above). The advantage to negative selection is it allows for the identification of new, large DNA insertions or mutations. Positive selection (like the SureSelect method described here) can enrich a target hundreds to thousands fold, making it possible to sequence low titer samples.

The positive enrichment approach described in this study shows a relatively simple and universal CLas genome enrichment method. We were able to efficiently get $99 \%$ coverage of the reference genome with over $70 \mathrm{X}$ sequence coverage using fewer than 5 million total reads even with a low to mid-titer pathogen sample (Cq value of 28.52). Thus this method makes large scale sequencing of the CLas genome more cost effective and applicable. More importantly, this method significantly pushes the sequencing limitation to much lower titer samples while preserving strain diversity. This is exemplified by the CLas genome of the lowest titer sample (equivalent to 28.52 Cq using Li 16S qPCR) being easily obtained with just 3.2 million total reads. Therefore, it could be possible to obtain the whole genome with even lower titer if more reads are used for the sample. Importantly, the RNA probe design of this positive capture method ensures retention of strain diversity, which other positive selection methods using primers run a risk of losing. This was exemplified by the phylogenetic analysis showing samples from two different locations clustering separately from one another (diversity retained), yet sequencing the same sample at different titer levels clustered together (reproducible results). These results indicate that this SureSelect target enrichment method can be used to sequence CLas more efficiently than the canonic NGS method. In the future, it will be interesting to determine the absolute sequencing limit of this method.

The most divergent region of the CLas genome is the prophage region, where strains can contain one to three prophages (or, in rare instances, none), with three known prophage types. Not surprisingly, we got the same prophage pattern for the SGCA strain sequenced in this study as SGCA5 (SC1 only), another strain from the same location ${ }^{14}$. Interestingly, LHCA contains both SC1 and SC2, meaning it has a different prophage profile and corresponds to the different clustering we observed in our phylogenetic analyses ${ }^{18}$ suggesting a potential different pathogen entry pathway. The probe set here use the SC1, SC2 and JXGC-3 as three prophage reference genomes, but we anticipate that it would capture all type 1, type 2 and type 3 prophage sequences if present in the samples. Although the mapping tracks show some different gaps among different strains suggesting uncovered non-conserved regions, the probes still capture sufficient prophage sequences for diversity analysis.

Besides the capability to sequencing medium to low titer samples, the total cost was also reduced by using SureSelect for the whole genome sequencing. Usually it costs at least \$1500 to \$3000dollars to whole genome sequence one high titer sample, but this was substantially reduced after using SureSelect target enrichment. In this study, it costs $\$ 500$ per sample to obtain the whole genome, which includes $\$ 300$ RNA probe per reaction and $\$ 200$ sequencing price. The RNA probe price can drop further to around $\$ 100$ dollar per sample if it is bulk order (96 reactions each order instead of 16 ). 
In summary, our data suggest that SureSelect-based target enrichment system is an excellent and cost effective method for CLas whole genome sequencing from infected citrus samples, including those with pathogen titer far lower than those used in previous studies.

\section{Material and Methods}

Custom capture library design. The SureSelect custom capture library was designed by Agilent. Probes were designed for the capture of DNA sequences from the "Candidatus Liberibacter asiaticus" listed on Table S1 including whole genome sequences of Ishi strain (no prophage sequences), SC1 prophage, SC2 prophage, JXGC-3 prophage and unique sequences from the other five CLas strains with complete genomes available on NCBI. Overall, 12620 RNA probes were designed. Each probe consists of 120 mer RNA and the total probe size is 1.32Mbp (Table S1).

Plant material and DNA extraction. Two CLas infected citrus branches containing LaHabra strain (LHCA) and San Gabriel strain (SGCA) were originally provided by California Department of Food and Agriculture (CDFA) and grafted to healthy citrus trees in the high containment green house of USDA APHIS PPQ Beltsville Laboratory. Successful grafted citrus trees were determined by HLBaspr real-time quantitative PCR from symptomatic leaves. CLas positive leaf samples from grafted trees were collected for genomic DNA extraction. Genomic DNA was extracted from petiole and leaf midrib tissue using the DNeasy Plant Mini Kit (Qiagen, Valencia, CA). The concentration of "Ca. Liberibacter asiaticus" was estimated using HLBaspr real-time quantitative PCR, giving a quantification threshold (Cq) value ${ }^{6}$. Four different Cq value $(20.1,22.84,26.84$, and 28.52) LHCA strain samples and two different Cq value (20.61 and 22.16) SGCA samples were selected to assess the sensitivity and selectivity of whole-genome enrichment and sequencing.

SureSelect XT HS target enrichment: library preparation, hybridization and enrichment. A total of $1 \mu \mathrm{g}$ input DNA per sample was used for SureSelect library preparation (Agilent, Santa Clara, CA). The library preparations were performed according to the SureSelect XT HS Target Enrichment System for Illumina Paired-End Multiplexed Sequencing Library protocol (Version A1, July 2017). The overall workflow is depicted in Fig. S1. First, all DNA samples were sheared using a M220 sonicator (Covaris, Woburn, MA) (duty factor 20\%, peak/Displayed Power (W) 50 and 200 cycles/burst for 30 second duration time), and adaptors were ligated to end repaired DNA. Adapter-ligated libraries were purified using AMPure XP beads (Beckman Coulter, Inc., Brea, CA, USA), amplified, and then purified. Quality and quantity of libraries were determined by TapeStation using a D1000 ScreenTape (Agilent). Next, $1 \mu \mathrm{g}$ of each library was hybridized with the SureSelect capture library. The hybridized libraries were purified with Dynabeads MyOne Streptavidin T1 magnetic beads (ThermoFisher Scientific, Waltham, MA), then the beads with captured DNA were washed one time with wash buffer 1 and five times with wash buffer 2 to remove non-specific binding. After all wash steps, the beads were suspended in $50 \mu \mathrm{l}$ of nuclease free water. Twenty-five $\mu \mathrm{l}$ of the DNA libraries, bound to streptavidin beads, was amplified by PCR using SureSelect post capture primer mix and Herculase II Fusing DNA polymerase. The cycling conditions were as follows: $98 \mathrm{C}$ for $2 \mathrm{~min}$; followed by $16-24$ cycles of $98 \mathrm{C}$ for $30 \mathrm{~s}, 60 \mathrm{C}$ for $30 \mathrm{~s}$, and $72 \mathrm{C}$ for $1 \mathrm{~min}$; and a final extension at $72 \mathrm{C}$ for $5 \mathrm{~min}$., using 16 cycles for Cq 20 samples, 18 cycles for Cq 22 samples, and 24 cycles for Cq 26 and Cq 28 samples. After PCR, streptavidin beads were removed using a magnet stand, and the PCR products were further purified with AMPure XP beads. High quality libraries were identified with an Agilent TapeStation using High Sensitivity D 1000 ScreenTape and then pooled for sequencing.

Illumina paired-end sequencing libraries preparation without target enrichment. We generated libraries for all six samples in parallel without enrichment using a TruSeq PCR free DNA library preparation kit (Illumina, San Diego, CA). A total of $2 \mu \mathrm{g}$ input DNA was fragmented using a Covaris M220 with the same setting as SureSelect enrichment library preparation.

Illumina sequencing. Sequencing of SureSelect enriched and non-enriched libraries was performed on an Illumina MiSeq platform (Illumina) on two separate v3 600 -cycle cartridges $(2 \times 300 \mathrm{bp})$. Base calling and sample de-multiplexing were generated as paired FASTQ files for each sample. All raw read files were deposited to the SRA public database under BioProject ID PRJNA540608.

Bioinformatics analysis. Read preprocessing. Raw reads were trimmed of adapter sequences and beginnings and ends trimmed where quality dropped to 0 . Reads were discarded with a mean quality score of less than 10 or when shorter than 200 base pairs, to avoid potential probe contamination, using BBDuk v38.12 (http://bbtools.jgi.doe.gov).

Prophage diversity. To determine the prophage content of each sample, we aligned all the reads from enriched samples to SC1, SC2 and JXGC3 prophage reference sequences using bowtie2 plugged in Geneious v 10.2.4 $4^{25}$, and visualized alignments in Integrated Genome Viewer v2.4.10 26,27 .

Genome alignment and SNP calling. Filtered high quality reads were mapped to the HLB Psy62 strain reference genome (GenBank accession number GCA_000023765.2) using bowtie2 v2.3.3 in sensitive mode ${ }^{23}$. Optical and PCR duplicates were flagged in alignment files using Picard v.2.10.5 (http://broadinstitute.github.io/ picard). Alignment files were filtered to remove PCR duplicates, retaining only reads in proper pairs with robust mapping quality (MAPQ $\geq 10$ ) using Samtools v. $1.7^{28}$. The cleaned alignment files were used to call single nucleotide polymorphisms (SNPs) with Samtools using the mpileup function, and SNP and indel genotypes in Variant Call Formatted (VCF) format were generated using BCFtools v1. $8^{26}$. VCF files were filtered to retain only variants sequenced to a minimum depth of coverage of 10 in enriched samples, and 3 in non-enriched samples. Shared and unique variants were compared within and between samples using vcftools "-diff-site" function. 
Phylogenetic methods. Phylogenies were generated with all samples and 11 published genomes (Table S2) using two methods, 'core SNPs' and the 'pan-genome'. Core SNPs were identified by mapping trimmed and filtered reads, as well as published genomes, against the Psy62 reference genome to create a whole genome alignment (including invariant sites), keeping sites with at least 10x coverage and greater than $90 \%$ consensus for each strain using Snippy v4.0 (https://github.com/tseemann/snippy). Genomic regions of high recombination were detected and removed with Gubbins v2.3.129, and filtered polymorphic sites extracted to build phylogenies. A total of 849 core SNPs were used to construct 10 maximum likelihood trees using a general time reversible model with gamma correction (GTRGAMMA) and 10,000 rapid bootstraps with RaxML v8.2.10 $0^{30}$. The tree with the highest likelihood across 10 runs was selected. The resulting tree was midpoint rooted and visualized using FigTree v1.4.3 (http://tree.bio.ed.ac.uk/software/figtree/).

For pan-genome generation, reads mapping to the Psy62 reference genome were extracted and assembled using SPAdes v3.12.0 with k-mer lengths of 21, 33, 55, 77, 99, and $127^{31}$. Contigs were reordered with Abacas v1.3. $1^{32}$ using the CLas strain Psy62 as a reference, and then annotated with Prokka v1.12 ${ }^{33}$. The annotated assemblies, as well as the 11 published genomes, were used to estimate the pan-genome with a 95\% Blast ID cutoff using Roary v3.12.0 $0^{34}$. Core alignments of 935 genes were extracted and used to estimate a maximum likelihood tree using RaxML, as outlined above.

Received: 21 May 2019; Accepted: 18 November 2019;

Published online: 12 December 2019

\section{References}

1. Jagoueix, S., Bové, J. M. \& Garnier, M. The phloem-limited bacterium of greening disease of citrus is a member of the $\alpha$ subdivision of the Proteobacteria. Int J Syst Bacteriol 44, 379-386 (1994).

2. Bové, J. M. Huanglongbing: a destructive, newly emerging, century-old disease of citrus. J Plant Pathol 88, 37-3714 (2006).

3. Teixeira Ddo, C. et al. Candidatus Liberibacter americanus, associated with citrus huanglongbing (greening disease) in São Paulo State, Brazil. Int J Syst Evol Microbiol. 55(Pt 5), 1857-62 (2005).

4. Gottwald, T.R, da Graça, J.V, \& Bassanezi, R.B. Citrus huanglongbing: the pathogen and its impact. Plant Health Progr, https://doi. org/10.1094/PHP-2007-0906-01-RV (2007).

5. Halbert, S. E. The discovery of huanglongbing in Florida. Proceedings of the 2nd International Citrus Canker and Huanglongbing Research Workshop 2005, Orlando Florida, USA, p50 (2005).

6. Li, W., Hartung, J. S. \& Levy, L. Quantitative real-time PCR for detection and identification of Candidatus Liberibacter species associated with citrus huanglongbing. J Microbiol Methods 66, 104-115 (2006).

7. Li., W., Levy, L. \& Hartung, J. S. Quantitative distribution of 'Candidatus Liberibacter asiaticus' in citrus plants with citrus huanglongbing. Phytopathology. 99(2), 139-44 (2009).

8. Puttamuk, T. et al. Genetic diversity of Candidatus Liberibacter asiaticus based on two hypervariable effector genes in Thailand. PLoS One, https://doi.org/10.1371/journal.pone.0112968 (2014).

9. Deng, X. et al. Characterization of "Candidatus Liberibacter asiaticus" populations by double-locus analyses. Curr Microbiol. 69(4), 554-60 (2014).

10. Ghosh, D. K. et al. Genetic Diversity of the Indian Populations of 'Candidatus Liberibacter asiaticus' Based on the Tandem Repeat Variability in a Genomic Locus. Phytopathology. 105(8), 1043-9 (2015).

11. Katoh, H. et al. Differentiation of "Candidatus Liberibacter asiaticus" isolates by variable-number tandem-repeat analysis. Appl Environ Microbiol. 77, 1910-1917 (2011).

12. Islam, M. S. et al. Multilocus microsatellite analysis of 'Candidatus Liberibacter asiaticus' associated with citrus Huanglongbing worldwide. BMC Microbiol. 20, 12-39 (2012).

13. Duan, Y. et al. Complete genome sequence of citrus huanglongbing bacterium, 'Candidatus Liberibacter asiaticus' obtained through metagenomics. Mol Plant Microbe Interact. 22, 1011-1020 (2009).

14. Wu, F. et al. Draft Genome Sequence of "Candidatus Liberibacter asiaticus" from a Citrus Tree in San Gabriel, California. Genome Announc. 3(6), https://doi.org/10.1128/genomeA.01508-15 (2015).

15. Kunta, M. et al. Draft whole-genome sequence of "Candidatus Liberibacter asiaticus" strain TX2351 isolated from Asian citrus psyllids in Texas, USA. Genome Announc, https://doi.org/10.1128/genomeA.00170-17 (2017).

16. Zheng, Z. et al. A Type 3 Prophage of 'Candidatus Liberibacter asiaticus' Carrying a Restriction-Modification System. Phytopathology. 108(4), 454-461, https://doi.org/10.1094/PHYTO-08-17-0282-R (2018).

17. Cai, W., Yan, Z., Rascoe, J. \& Stulberg, M. J. Draft Whole-Genome Sequence of “Candidatus Liberibacter asiaticus" Strain TX1712 from Citrus in Texas. Genome Announc. 6(25), https://doi.org/10.1128/genomeA.00554-18 (2018).

18. Dai, Z. et al. Prophage Diversity of "Candidatus Liberibacter asiaticus" Strains in California. Phytopathology, https://doi.org/10.1094/ PHYTO-06-18-0185-R (2018).

19. Gnirke, A. et al. Solution hybrid selection with ultra-long oligonucleotides for massively parallel targeted sequencing. Nat Biotechnol 27, 182-189 (2009).

20. Mamanova, L. et al. Target-enrichment strategies for next-generation sequencing. Nat Methods. 7(2), 111-8 (2010).

21. Schuenemann, V. J. et al. Targeted enrichment of ancient pathogens yielding the pPCP1 plasmid of Yersinia pestis from victims of the Black Death. Proc Natl Acad Sci USA 108, E746-752 (2011).

22. Wylie, T. N., Wylie, K. M., Herter, B. N. \& Storch, G. A. Enhanced virome sequencing using targeted sequence capture. Genome Res. 25, 1910-1920 (2015).

23. Clark, S. A., Doyle, R., Lucidarme, J., Borrow, R. \& Breuer, J. Targeted DNA enrichment and whole genome sequencing of Neisseria meningitidis directly from clinical specimens. Int J Med Microbiol. 308(2), 256-262 (2018).

24. Zheng, Z., Deng, X., \& Chen, J. Draft Genome Sequence of “Candidatus Liberibacter asiaticus" from California. Genome Announc, https://doi.org/10.1128/genomeA.00999-14 (2014).

25. Langmead, B. \& Salzberg, S. L. Fast gapped-read alignment with Bowtie 2. Nat Methods. 9, 357-359 (2012).

26. Robinson, J. T. et al. Mesirov. Integrative Genomics Viewer. Nature Biotechnology. 29, 24-26 (2011).

27. Thorvaldsdóttir, H., Robinson, J. T. \& Mesirov, J. P. Integrative Genomics Viewer (IGV): high-performance genomics data visualization and exploration. Briefings in Bioinformatics. 14, 178-192 (2013).

28. Li, H. A statistical framework for SNP calling, mutation discovery, association mapping and population genetical parameter estimation from sequencing data. Bioinformatics 27(21), 2987-2993 (2011).

29. Croucher, N. J. et al. Rapid phylogenetic analysis of large samples of recombinant bacterial whole genome sequences using Gubbins. Nucleic acids research. 43(3), e15-e15 (2014).

30. Stamatakis, A. RAxML version 8: a tool for phylogenetic analysis and post-analysis of large phylogenies. Bioinformatics. 30(9), $1312-1313$ (2014). 
31. Bankevich, A. et al. SPAdes: a new genome assembly algorithm and its applications to single-cell sequencing. Journal of computational biology. 19(5), 455-477 (2012).

32. Assefa, S., Keane, T. M., Otto, T. D., Newbold, C. \& Berriman, M. ABACAS: algorithm-based automatic contiguation of assembled sequences. Bioinformatics. 25(15), 1968-1969 (2009).

33. Seemann, T. Prokka: rapid prokaryotic genome annotation. Bioinformatics. 30(14), 2068-2069 (2014).

34. Page, A. J. et al. Roary: rapid large-scale prokaryote pan genome analysis. Bioinformatics. 31(22), 3691-3693 (2015).

\section{Acknowledgements}

We thank California Department of Food and Agriculture (CDFA) for providing the infected citrus samples. This research was supported by the intramural research and citrus health response programs of the U.S. Department of Agriculture, Animal and Plant Health Inspection Service. The findings and conclusions in this publication are those of the authors and should not be construed to represent any official USDA or U.S. Government determination or policy.

\section{Author contributions}

M.S. and W.C., Conceived and designed the experiments. W.C., conducted the experiments. S.N. and W.C., collected and analyzed data. W.C., S.N., J.R. and M.S., wrote and revised the manuscript. All authors reviewed and approved the manuscript.

\section{Competing interests}

The authors declare no competing interests.

\section{Additional information}

Supplementary information is available for this paper at https://doi.org/10.1038/s41598-019-55144-4.

Correspondence and requests for materials should be addressed to M.J.S.

Reprints and permissions information is available at www.nature.com/reprints.

Publisher's note Springer Nature remains neutral with regard to jurisdictional claims in published maps and institutional affiliations.

(1) Open Access This article is licensed under a Creative Commons Attribution 4.0 International License, which permits use, sharing, adaptation, distribution and reproduction in any medium or format, as long as you give appropriate credit to the original author(s) and the source, provide a link to the Creative Commons license, and indicate if changes were made. The images or other third party material in this article are included in the article's Creative Commons license, unless indicated otherwise in a credit line to the material. If material is not included in the article's Creative Commons license and your intended use is not permitted by statutory regulation or exceeds the permitted use, you will need to obtain permission directly from the copyright holder. To view a copy of this license, visit http://creativecommons.org/licenses/by/4.0/.

(c) The Author(s) 2019 